\title{
Genetic diversity and drug resistance among newly diagnosed and antiretroviral treatment- naive HIV-infected individuals in western Yunnan: a hot area of viral recombination in China
}

Min Chen ${ }^{1+}$, Yanling Ma ${ }^{1+}$, Song Duan², Hui Xing ${ }^{3}$, Shitang Yao ${ }^{2}$, Yingzhen Su', Hongbing Luo ${ }^{1}$, Li Yang ${ }^{1}$,

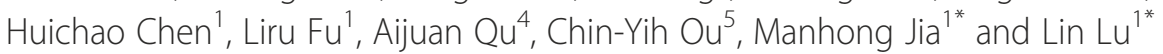

\begin{abstract}
Background: The emergence of an HIV-1 epidemic in China was first recognized in Dehong, western Yunnan. Due to its geographic location, Dehong contributed greatly in bridging HIV-1 epidemics in Southeast Asia and China through drug trafficking and injection drug use; and also extensively to the HIV genetic diversity in Yunnan and China. We attempt to monitor HIV-1 in this area by studying the HIV-1 genetic distribution and transmitted drug resistance (TDR) in various at-risk populations.

Methods: Blood samples from a total of 320 newly HIV-1 diagnosed individuals, who were antiretroviral therapy (ART)-naive, were collected from January 2009 to December 2010 in 2 counties in Dehong. HIV-1 subtypes and pol gene drug resistance (DR) mutations were genotyped.

Results: Among 299 pol sequences successfully genotyped (93.4\%), subtype $C$ accounted for $43.1 \%$ ( $n=129$ ), unique recombinant forms (URFs) for 18.4\% ( $n=55)$, CRF01_AE for $17.7 \%(n=54)$, B for 10.7\% ( $n=32)$, CRF08_BC for $8.4 \%$ $(n=25)$ and CRF07_BC for 1.7\% ( $n=5)$. Subtype distribution in patients infected by different transmission routes varied. In contract to the previous finding of CRF01_AE predominance in 2002-2006, subtype C predominated in both injecting drug users (IDUs) and heterosexually transmitted populations in this study. Furthermore, we found a high level of BC, CRF01_AE/C and CRF01_AE/B/C recombinants suggesting the presence of active viral recombination in the area. TDR associated mutations were identified in $4.3 \%(n=13)$ individuals. A total of $1.3 \%$ of DR were related to protease inhibitors (PIs), including I85IV, M46I and L90M; 0.3\% to nucleoside reverse transcriptase inhibitors (NRTIs), including M184l; and 2.7\% to non-nucleoside reverse transcriptase inhibitors (NNRTIs), including K103N/S, Y181C, K101E and G190A.

Conclusion: Our work revealed diverse HIV-1 subtype distributions and intersubtype recombinations. We also identified a low but significant TDR mutation rate among ART-naive patients. These findings enhance our understanding of HIV-1 evolution and are valuable for the development and implementation of a comprehensive public health approach to HIV-1 DR prevention and treatment in the region.
\end{abstract}

Keywords: HIV-1, Genetic diversity, Drug resistance, Injecting drug use, Dehong, China

\footnotetext{
* Correspondence: jmanhong@yahoo.com.cn; lulin@yncdc.cn

${ }^{\dagger}$ Equal contributors

'Yunnan Center for AIDS/STD Control and Prevention, Yunnan Center for

Disease Control and Prevention, Kunming, Yunnan 650022, China

Full list of author information is available at the end of the article
} 


\section{Background}

Dehong prefecture is located in western Yunnan bordering northern Myanmar. It is in a key position along the drug trafficking routes from Southeast Asia's opiumproducing and high HIV prevalent "Golden Triangle" region into China [1]. The first HIV epidemic in China with the identification of 146 infected IDUs was reported in Dehong in 1989 [2]. To date, Dehong remains one of the most HIV-affected areas in China [3,4].

Subtype B and subtype B' (the B subtype originally found in Thailand) were the HIV-1 strains first found among infected Dehong IDUs [5-7]. The proportion of subtype $\mathrm{B}^{\prime}$ among the subtype B category increased from 20\% in 1990 to $90 \%$ in 1996 [5-7]. Subtype C was identified in IDUs from India in the early 1990s [8] and cocirculated with $\mathrm{B}^{\prime}$ in the IDUs in western Yunnan in the first half of the 1990s resulting in many recombinant viruses [9-12]. Another CRF01_AE subtype was also found in this region in 1994 [13] and, with increased sexual contacts, became the predominant strain in 2000 $[9,10]$. Other viral strains, CRF07_BC and CRF08_BC, were also found in Dehong in 2002 [9,10,12].

To combat the growing HIV infection, China initiated a national free ART program with standardized treatment options and management protocols in 2003 and by the end of 2010, the total number of ART patients exceeded 100,000. Although the widespread use of highly active ART successfully decreases HIV/AIDSrelated mortality and morbidity [14,15], suboptimal ART adherence may accelerate the emergence of drug resistant viruses [16] and compromise ART efficacy in treated individuals and transmission recipients. It was reported that suboptimal ART management in central China resulted in the rise of DR rate from $13.9 \%, 45.4 \%$ to $62.7 \%$ in individuals who were ART-naive and 3- and 6months post-ART, respectively [17]. By the end of 2010, of the 19,512 ART patients in Yunnan, 20\% resided in Dehong. It is important to conduct HIV DR surveys to estimate the TDR rate among ART-naive populations with different transmission routes to provide baseline information for the formulation of effective ART regimens to delay the emergence of DR and to develop a rational public health strategy to control the HIV epidemic.

\section{Methods}

\section{Study area, population and sampling}

Blood samples were collected from 320 individuals newly diagnosed with HIV-1 infection in Longchuang and Luxi counties of Dehong prefecture between January 2009 and December 2010. HIV-1 infection was confirmed by Western Blot assay (HIV BLOT 2.2, MP Diagnostics, Singapore). All study participants were ART-naive and provided informed consent. This study was approved by the institutional review board of Yunnan Center for Disease Control and Prevention.

\section{Amplification and sequencing of HIV-1 pol}

Viral RNA was extracted from $200 \mu \mathrm{l}$ plasma using an automated extractor (MagNA Pure LC system, Roche, Branchburg, NJ) according to the manufacturer's instructions. For the amplification of the pol regions, an in-house nested reverse transcription PCR method was used. The target sequence was amplified with One Step Reverse Transcription PCR reagents procured from Takara (Dalian, China) using primers MAW26 (5'-TTG GAAATGTGGAAAGGAAGGAC-3') and RT21 (5'-CT GTATTTCTG CTATTAAGTCTTTTGATGGG-3') in a $25 \mu \mathrm{l}$ reaction for 30 cycles. Cycling conditions were $50^{\circ} \mathrm{C}$ for $30 \mathrm{~min}, 94^{\circ} \mathrm{C}$ for $5 \mathrm{~min}, 94^{\circ} \mathrm{C}$ for $30 \mathrm{~s}, 55^{\circ} \mathrm{C}$ for $30 \mathrm{~s}$, $72^{\circ} \mathrm{C}$ for $2.5 \mathrm{~min}$, followed with an extension at $72^{\circ} \mathrm{C}$ for $10 \mathrm{~min}$. The nested PCR was performed using Taq PCR master mix (Tiangen, Beijing, China), using primers PRO1 (5'-CAGAGCCAACAGCCCCACCA-3') and RT20 (5'CTGCCAGTTCTAGCTCTGCTTC-3') in a $50 \mu \mathrm{l}$ reaction for 30 cycles and the cycling conditions were $94^{\circ} \mathrm{C}$ for 5 min, $94^{\circ} \mathrm{C}$ for $30 \mathrm{~s}, 63^{\circ} \mathrm{C}$ for $30 \mathrm{~s}, 72^{\circ} \mathrm{C} 2.5 \mathrm{~min}$, followed with an extension at $72^{\circ} \mathrm{C}$ for $10 \mathrm{~min}$. The resulting PCR product of $1191 \mathrm{bp}$ in length contained a full length protease (PR) gene of 99 amino acid codons and the first 298codon segment of the reverse transcriptase (RT) gene. PCR products were visualized by $1 \%$ agarose gel electrophoresis. Successfully amplified samples were sequenced by Biomed Co. (Beijing, China) on an ABI 3730xl automated DNA analyzer (Applied Biosystems, Foster City, CA) with primers: PROS3 (5'-GCCAACAGCCCCACCA-3'), RTAS (5'-CTCAGATTGGTTGCAC-3'), RTB (5'-CCTAGTA TAAACAATGAGACAC-3'), PROC1S (5'-GCTGGGTGT GGTATTCC-3') and RT20S3 (5'-GTTCTAGCTCTGCT TC-3'). Each step was carried out with appropriate negative controls to detect PCR-related contamination during the experiments.

\section{Sequence analysis}

The sequence contig assembly was performed using analysis software, Sequencher 4.9 (Gene Codes Corporation, Ann Arbor, MI). The ClustalW Multiple alignment and manual editing were performed using BioEdit Sequence Alignment Editor (Ibis Biosciences, Carlsbad, CA). Edited sequences were evaluated for DR mutations using both the Surveillance Drug Resistance Mutations (SDRM) list recommended by the World Health Organization [18], and the Stanford University Algorithm (http://hivdb.stanford.edu). The HIV-1 subtypes of pol sequences were identified by the REGA HIV subtyping tool (http://www.bioafrica.net/subtypetool/html/) [19]. To demonstrate possible intersubtype mosaicism, candidate sequences were analyzed using the Recombination 
Identification Program (RIP; version 3.5.1) available at the HIV sequence database site (http://hiv-web.lanl.gov). Bootscanning analysis was performed with default parameters of window: 200bp, step: 20bp, gapsrip: on, reps: 100, Kinura (2-parameter), T/t: 2.0. Similarity plot analysis (SimPlot version 3.5.1; S. Ray, Johns Hopkins University, Baltimore, MD; http://sray.med.som.jhmi.edu/RaySoft/SimPlot/) was performed using reference A1, B, C, CRF07_BC, CRF08_BC and CRF01_AE strains. All sequences obtained in this study were submitted to GenBank with accession numbers from JQ658474 to JQ658772.

\section{Statistical analysis}

Statistical analysis was conducted using the SPSS 17.0 package (SPSS Inc. Chicago, IL). Categorical variables were compared using chi-square analysis. When the theoretical frequency was less than 5, Fisher exact test was used. All tests were two-tailed and a $p$-value $<0.05$ was considered significant.

\section{Results}

Demographic characteristics of patients

A total of 320 individuals diagnosed with HIV-1 infection between 2009 and 2010 were recruited from two counties in Dehong prefecture. Two hundred ninety nine sequences (93.4\%) of HIV-1 pol region were successfully amplified and sequenced. The demographics of these individuals are shown in Table 1 . The ratio of males to females was 1:0.78 and the mean age was 32.5 years (range: 2-74 years). Heterosexual transmission

Table 1 Demographic characteristics and HIV DR of study individuals

\begin{tabular}{|c|c|c|c|c|}
\hline \multirow[b]{2}{*}{ Total } & \multicolumn{2}{|c|}{ Total N (\%) } & \multirow{2}{*}{$\begin{array}{l}\text { No. of resistant mutations } \\
13\end{array}$} & \multirow{2}{*}{$\begin{array}{l}\text { Resistance rate (\%) } \\
4.5\end{array}$} \\
\hline & 299 & $(100)$ & & \\
\hline \multicolumn{5}{|l|}{ Gender } \\
\hline Male & 168 & $(56.2)$ & 7 & 4.3 \\
\hline Female & 131 & $(43.8)$ & 6 & 4.8 \\
\hline \multicolumn{5}{|l|}{ Age } \\
\hline$\leq 25$ & 75 & $(25.1)$ & 3 & 4.2 \\
\hline $26-35$ & 130 & $(43.5)$ & 6 & 4.8 \\
\hline $36-45$ & 58 & $(19.4)$ & 4 & 7.4 \\
\hline$>45$ & 36 & $(12.0)$ & 0 & 0 \\
\hline \multicolumn{5}{|l|}{ Transmission routes } \\
\hline Heterosexual & 227 & $(75.9)$ & 8 & 3.7 \\
\hline Intravenous drug injection & 52 & $(17.4)$ & 5 & 10.6 \\
\hline Homosexual & 2 & $(0.7)$ & 0 & 0 \\
\hline Mother to child & 4 & (1.3) & 0 & 0 \\
\hline Unknown & 14 & $(4.7)$ & 0 & 0 \\
\hline \multicolumn{5}{|l|}{ Ethnicity } \\
\hline Han & 122 & $(40.8)$ & 3 & 2.5 \\
\hline Jingpo & 98 & $(32.8)$ & 7 & 7.7 \\
\hline Dai & 64 & $(21.4)$ & 2 & 3.2 \\
\hline Others & 15 & $(5.0)$ & 1 & 7.1 \\
\hline \multicolumn{5}{|l|}{ CD4 $^{+}$Count $($cells $/ \mu l)$} \\
\hline$\leq 200$ & 21 & $(7.0)$ & 1 & 5.0 \\
\hline $201-350$ & 64 & $(21.4)$ & 3 & 4.9 \\
\hline $351-500$ & 88 & $(29.4)$ & 4 & 4.8 \\
\hline$\geq 501$ & 126 & $(42.1)$ & 5 & 4.1 \\
\hline \multicolumn{5}{|l|}{ HIV-1 Subtype } \\
\hline C & 129 & $(43.1)$ & 7 & 5.7 \\
\hline URFs & 55 & $(18.4)$ & 2 & 3.8 \\
\hline CRF01_AE & 53 & $(17.7)$ & 1 & 1.9 \\
\hline B & 32 & $(10.7)$ & 2 & 6.7 \\
\hline CRF08_BC & 25 & $(8.4)$ & 1 & 4.2 \\
\hline CRF07_BC & 5 & (1.7) & 0 & 0.0 \\
\hline
\end{tabular}

Abbriviations: URFs, unique recombinant forms; CRFs, circulating recombinant forms. 
constituted the most frequent transmission route $(75.9 \%$, $\mathrm{n}=227$ ), followed by intravenous drug injection $(17.4 \%$, $\mathrm{n}=52)$, mother-to-infant transmission $(1.3 \%, \mathrm{n}=4)$ and homosexual transmission $(0.7 \%, \mathrm{n}=2)$. The transmission route of the remaining $4.7 \%(n=14)$ individuals was unknown. The ethnicities of the studied individuals were Han (40.8\%, $n=122)$, Jingpo (32.8\%, $n=98)$, Dai (21.4\%, $\mathrm{n}=64)$, Achang (2.3\%, $\mathrm{n}=7)$, Deang (1.3\%, $\mathrm{n}=4)$, Bai $(1.0 \%, \mathrm{n}=3)$, and Dongxiang $(0.3 \%, \mathrm{n}=1)$. There were also 10 Burmese individuals. In addition, $28.4 \%(n=85)$ of the individuals had CD4 cell count lower than 350 cells/ $\mu \mathrm{l}$ and were ART-eligible.

\section{HIV-1 subtypes distribution}

HIV-1 pol gene sequences (1191 bp) were subjected to subtype determination [20]. As revealed in Table 1, 6 basic subtypes were identified. HIV-1 C subtype was found to be the most common subtype $(43.1 \%, \mathrm{n}=129)$, followed by URFs (18.4\%, $\mathrm{n}=55), \mathrm{CRF} 01 \_\mathrm{AE}(17.7 \%$, $\mathrm{n}=53), B(10.7 \%, \mathrm{n}=32), \mathrm{CRF} 08 \_\mathrm{BC}(8.4 \%, \mathrm{n}=25)$ and CRF07_BC $(1.7 \%, \mathrm{n}=5)$. Bootscanning analysis revealed the presence of 55 (18.4\%) URFs including $48 \mathrm{BC}$ recombinants (16.1\%, Figure $1 \mathrm{C}$ and D), 6 CRF_01AE/C (2.0\%, Figure 1E-I) and 1 CRF_01AE/B/C (0.3\%, Figure 1J). The prevalence of URF (18.4\%) was similar to that of CRF01_AE (17.7\%) and revealed frequent recombination among the main strains (subtype C, CRF01_AE and subtype B). Most URFs $(76.4 \%, 42 / 55)$ were found in individuals infected through heterosexual contact (Table 2). Seven of the HIV sequences derived from the 10 Burmese individuals were subtype $C$ and the other 3 were URF_BC.

The presence of subtypes/CRFs varied with different transmission routes. As shown in Table 2, all 6 genotypes were found in the heterosexually transmitted population. Five genotypes, with the exception of CRF07_BC, were detected in IDUs. One subtype C, 1 URF and 2 CRF01_AE strains were detected in the 4 young children infected through mother-to-child transmission; 1 CRF01_AE strain and 1 CRF07_BC strain in 2 persons who were men having sex with men. The proportions of HIV-1 genotypes in heterosexually transmitted population and IDUs showed a statistical difference $\left(X^{2}=13.801, p=0.013\right)$ suggesting that different subtype distribution patterns were associated with different infection routes. The proportion of subtype $\mathrm{C}$ among IDUs (61.5\%) was significantly higher than that among the heterosexually transmitted population $\left(39.2 \%, X^{2}=8.591\right.$, $p=0.005)$. Likewise, the proportion of CRF01_AE in the heterosexually transmitted population (18.9\%) was
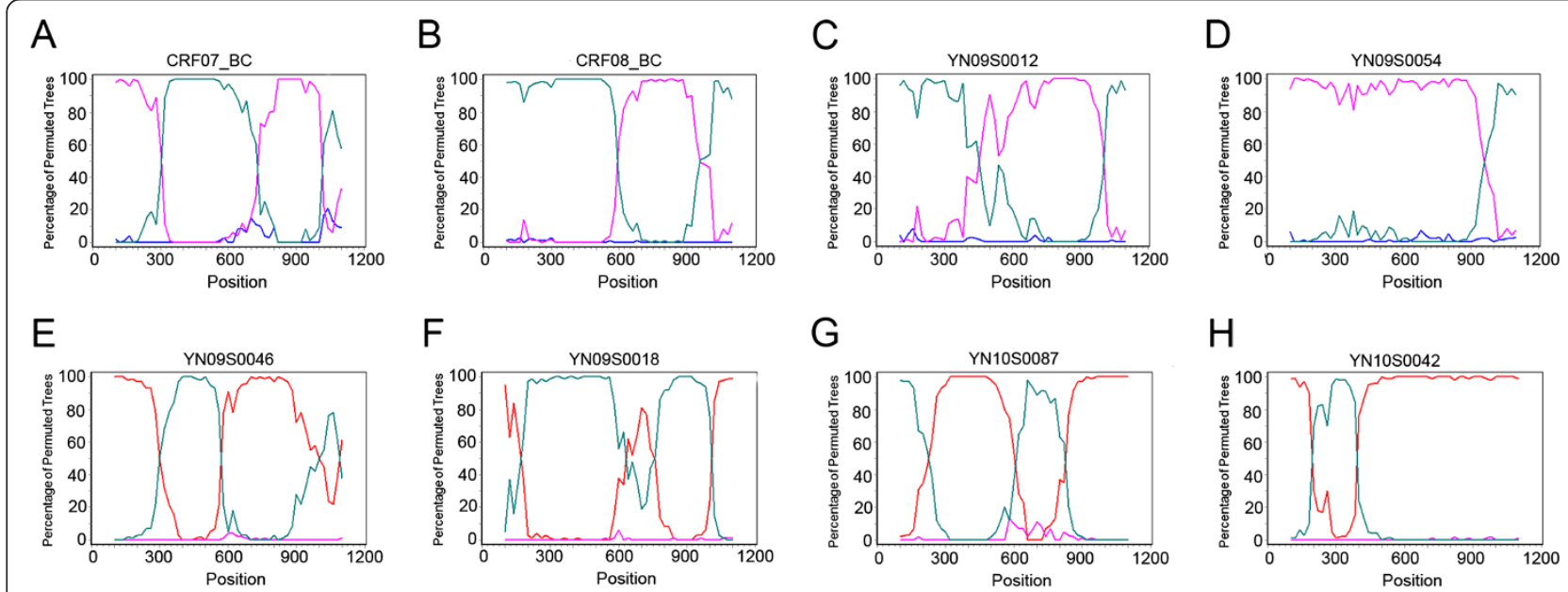

F

G
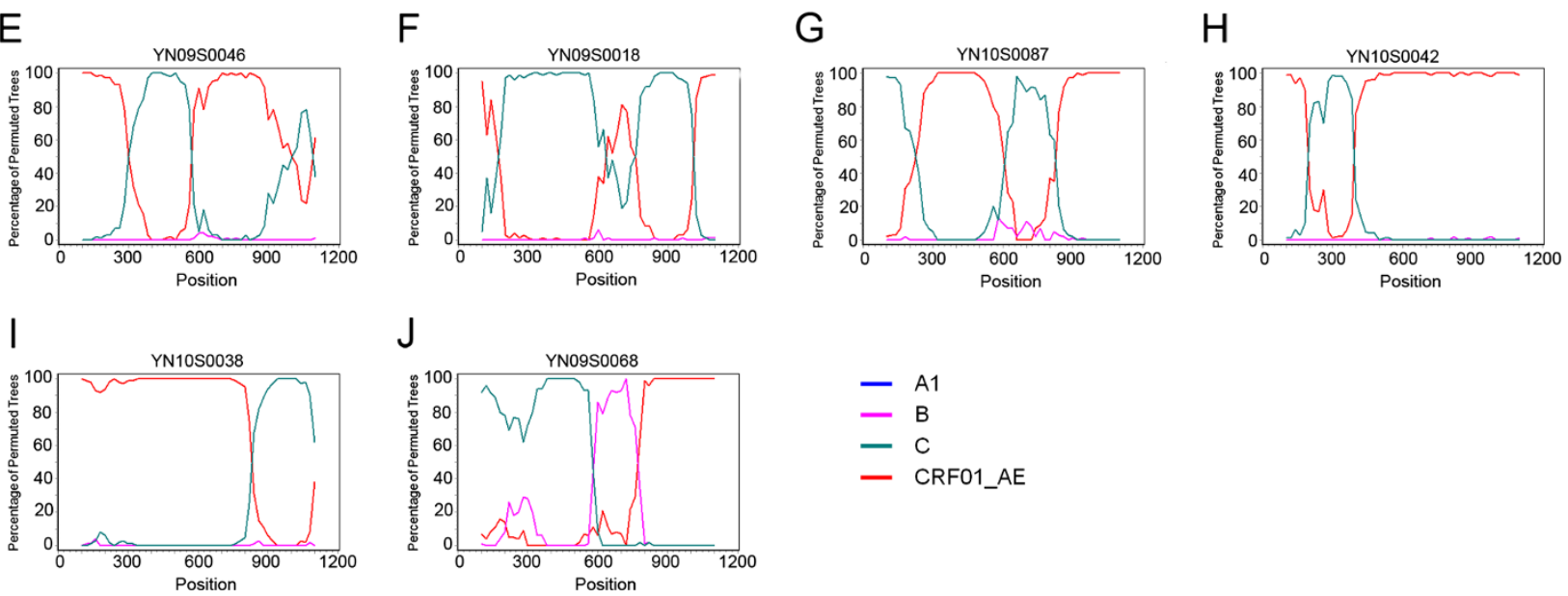

Figure 1 Bootscanning analysis of new viral recombination in HIV-1 pol gene region. (A) the reference sequence of CRF07_BC; (B) the reference sequence of CRF08_BC; (C) representative BC recombinant YN09S0012; (D) representative BC recombinant, YN09S0054; (E to I), representative CRF_01AE/C recombinant, E: YN09S0046, F: YN10S0018, G: YN10S0087, H: YN10S0042 and I: YN10S0038; and (J) representative CRF_01AE/B/C recombinant, YN09S0068. The conditions used for this analysis were following: Window: 200 bp, step: 20 bp, gapsrip: on, reps: 100, Kinura (2-parameter), T/t: 2.0. 
Table 2 The constitution of HIV-1 subtypes in different transmission routes

\begin{tabular}{|c|c|c|c|c|c|c|c|c|c|}
\hline Transmission route & Subjects & $\mathrm{C}$ & URFs & CRF01_AE & B & CRF08_BC & CRF07_BC & $x^{2}$ & $P^{*}$ \\
\hline Heterosexual & $227(100 \%)$ & 89 (39.2\%) & $42(18.5 \%)$ & $43(18.9 \%)$ & $25(11.0 \%)$ & $24(10.6 \%)$ & $4(1.8 \%)$ & 13.801 & 0.013 \\
\hline Intravenous drug injection & $52(100 \%)$ & $32(61.5 \%)$ & $9(17.3 \%)$ & $3(5.8 \%)$ & $7(13.5 \%)$ & $1(1.9 \%)$ & $0(0 \%)$ & & \\
\hline Homosexual & $2(100 \%)$ & $0(0 \%)$ & $0(0 \%)$ & $1(50.0 \%)$ & $0(0 \%)$ & $0(0 \%)$ & $1(50.0 \%)$ & & \\
\hline Mother to child & $4(100 \%)$ & $1(25.0 \%)$ & $1(25.0 \%)$ & $2(50.0 \%)$ & $0(0 \%)$ & $0(0 \%)$ & $0(0 \%)$ & & \\
\hline Unknown & $14(100 \%)$ & $7(50.0 \%)$ & $3(21.4 \%)$ & $4(28.6 \%)$ & $0(0 \%)$ & $0(0 \%)$ & $0(0 \%)$ & & \\
\hline
\end{tabular}

*: Compared the proportions of subtypes between groups of heterosexual contact and intravenous drug injection.

significantly higher than that among IDUs $\left(5.8 \%, \chi^{2}=5.332\right.$, $p=0.022)$. The proportions of URFs, subtype $\mathrm{B}$ and CRF08_BC showed no statistical difference in these two populations.

\section{Genotypic analysis of DR}

To detect TDR-associated mutations, the pol sequences were screened against those within the Stanford HIV Drug Resistance Database (http://hivdb.stanford.edu). DR-related mutations were identified in 13 of the 299 viral sequences $(4.3 \%)$. With the exception of one individual who had 2 NNRTI mutations, the other 12 individual harbored virus with 1 DR mutation each. The demographic characteristics of these 13 ART-naive individuals are shown in Table 3 . The mean age was 32.5 years. The mean CD4 cell count was 467 cells/ $\mu \mathrm{l}$ and 4 individuals were ART eligible. No statistical difference was observed in distribution of DR strains by gender, age, infection route, ethnicity, CD4 cell count or HIV-1 subtype. Eight were infected through heterosexual transmission and the other 5 through intravenous drug injection. One individual (YN10S0137) was Burmese.

The proportion of sequences with TDR mutation associated with NRTIs, NNRTIs, and PIs was $0.3 \%(n=1)$,
$2.7 \%(n=8)$, and $1.3 \%(n=4)$, respectively. Sequences containing mutations associated with 2 or 3 classes of antiretroviral drugs were not found. The PI-related DR included: I85V (0.67\%, $\mathrm{n}=2), \mathrm{M} 46 \mathrm{I}(0.33 \%, \mathrm{n}=1)$ and L90M $(0.33 \%, n=1)$. Although I85V is a nonpolymorphic PI-selected mutation, it does not result in resistance to PI. Only 1 NRTI-related DR mutation, M184I (0.33\%, $n=1)$, was detected. Among NNRTIrelated DR mutations, K103N and Y181C were found in 3 samples (1.0\%). K103N causes high-level resistance to nevirapine (NVP) and efavirenz (EFV). Y181C causes high-level resistance to NVP and intermediate resistance to EFV, etravirine (ETR) and rilpivirine (RPV). Importantly, Y181C forms the foundation for high-level ETR and RPV resistance as an addition of some single mutations and many double mutations causes high-level resistance to these drugs. Three individuals were found to have one other NNRTI-related mutation (K103NS, K101E and G190A) each (0.33\%).

Based on the interpretation of the Stanford HIV Drug Resistance Database, the 13 drug resistant viruses showed different levels of resistance to 11 antiretroviral drugs (Figure 2). Although lopinavir (LPV) and indinavir (IDV) were the main PIs used in Dehong, low-level resistance to

Table 3 Demographic characteristics of 13 individuals infected by viruses containing DR mutations

\begin{tabular}{|c|c|c|c|c|c|c|c|c|c|}
\hline \multirow{2}{*}{$\begin{array}{l}\text { Sequence } \\
\text { ID }\end{array}$} & \multirow[t]{2}{*}{ Gender } & \multirow[t]{2}{*}{ Age } & \multirow[t]{2}{*}{ Transmission route } & \multirow{2}{*}{$\begin{array}{l}\text { Ethnicity/ } \\
\text { Nationality }\end{array}$} & \multirow{2}{*}{$\begin{array}{l}\text { CD4 }^{+} \\
\text {Count } / \mu \mathrm{l}\end{array}$} & \multicolumn{3}{|c|}{ DR Mutation } & \multirow{2}{*}{$\begin{array}{l}\text { HIV-1 } \\
\text { Subtype }\end{array}$} \\
\hline & & & & & & NRTI & NNRTI & PI & \\
\hline YN09S0004 & Male & 37 & Intravenous drug injection & Jingpo & 383 & $-^{*}$ & $\mathrm{~K} 103 \mathrm{~N}$ & - & $\mathrm{B}$ \\
\hline YN09S0012 & Male & 55 & Heterosexual & Han & 506 & - & - & M46I & $B C$ \\
\hline YN09S0021 & Female & 42 & Heterosexual & Jingpo & 149 & - & K103N & - & CRF01_AE \\
\hline YN09S0027 & Female & 29 & Heterosexual & Achang & 225 & - & K101E & - & C \\
\hline YN09S0054 & Female & 38 & Heterosexual & Jingpo & 280 & - & K103N, Y181C & - & $\mathrm{BC}$ \\
\hline YN09S0072 & Female & 31 & Heterosexual & Jingpo & 422 & - & K103NS & - & B \\
\hline YN09S0092 & Female & 19 & Intravenous drug injection & Han & 335 & - & Y181C & - & C \\
\hline YN09S0114 & Male & 24 & Heterosexual & Jingpo & 882 & - & G190A & - & C \\
\hline YN09S0129 & Male & 25 & Heterosexual & Jingpo & 608 & - & - & L90M & CRF08_BC \\
\hline YN10S0097 & Male & 34 & Intravenous drug injection & Han & 639 & - & - & $185 \mathrm{~V}$ & C \\
\hline YN10S0132 & Male & 33 & Intravenous drug injection & Dai & 475 & - & Y181C & - & C \\
\hline YN10S0137 & Female & 22 & Heterosexual & Burmese & 473 & M184I & - & - & C \\
\hline YN10S0138 & Male & 33 & Intravenous drug injection & Jingpo & 687 & - & - & $185 \mathrm{~V}$ & $C$ \\
\hline
\end{tabular}

*: not detected. 
atazanavir (ATV), fosamprenavir (FPV), IDV, nelfinavir (NFV) and saquinavir (SQV) was detected. Among RTIs, intermediate and high-level resistance to 4 NNRTIs (EFV, ETR, NVP and RPV) and high-level resistance to 2 NRTIs (lamivudine (3TC) and emtricitabine (FTC)) were detected, of which 2 NNRTIs (ETR and RPV) and 1 NRTI (FTC) were not used in local patients. The reason for their presence might be due to cross-resistance, for example 3TC induced M184I/V but also rendered resistance to FTC. Further, cross-resistance was a common phenomenon among NNRTIs. The existence of cross-resistance could increase the complexity and the length of ART.

\section{Discussion}

Yunnan has been described as a "key HIV epicenter in China" [21]. By the end of 2010, the cumulative number of HIV/AIDS cases reported in Yunnan was 83,925 and accounted for $21 \%$ of total HIV/AIDS cases in China. With the scale up of ART in Yunnan, the emergence of HIV DR is anticipated. The surveillance of emergence and transmission of drug resistant HIV-1 strains is one of the priorities for HIV/AIDS prevention and control in Yunnan. In particular, surveying the characteristics and trends of TDR among newly reported cases of HIV infection could provide valuable information for evaluating the efficacy of ART, public health planning [22], and the selection of pre- and post-exposure prophylaxis.

By using a standard genotyping protocol, we carried out a survey of TDR in 299 newly HIV-1 diagnosed and ART-naive individuals residing in Dehong. Our study revealed a low TDR rate of $4.3 \%$. An earlier study conducted in 2006 of 64 newly infected HIV-1 patients whose infections were classified to be recent by BED incidence assay yielded a rate of $6.3 \%$ [23]. No statistical difference between the rates of these 2 studies $(p>0.05)$ was found. This indicates that the prevalence of drug resistant HIV-1 strains remained relatively constant over the 5 years between 2006 and 2010, and that the management of treated individuals with standardized ART protocol appeared to be efficient in western Yunnan. In this study, we also found one DR strain among 10 Burmese individuals. Further vigilance needs to be placed to monitor the importation of DR strains from neighboring countries.

The first-line regimens of the Chinese national free ART program include 2 NRTIs and 1 NNRTI. The ART drugs presently used in Dehong include 6 NRTIs (abacavir $(\mathrm{ABC})$, zidovudine $(\mathrm{AZT})$, stavudine $(\mathrm{D} 4 \mathrm{~T})$, didanosine (DDI), tenofovir (TDF) and 3TC, two NNRTIs (EFV and NVP), and two PIs (LPV and IDV). A total of $69.2 \%$ of drug resistant strains found in this study were RTIassociated. These DR mutations (M184I, K103N/S, Y181C and K101E) could derive from ART-treated patients in Yunnan [24]. Further, intermediate and highlevel resistance to four NNRTIs was detected with increased frequency. The increased rates of RTI relevant resistance strains could increase the failure of the firstline antiretroviral regimens. The use of PIs is limited and PI-related DR mutations were not previously found among the treated population in 2008 in Yunnan [24]. Five PI-related DR strains were detected in this study. A possible explanation for the presence of DR to PIs different from the ones used in Dehong (LPV and IDV) is that the individuals were administering self-procured PIs from overseas or the observed mutations were introduced by foreigners.

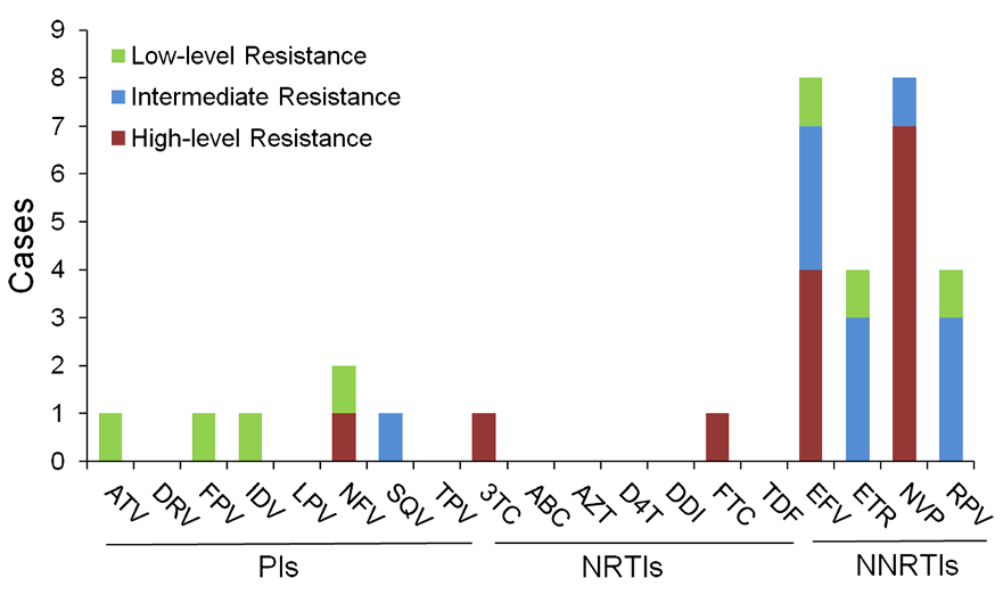

Figure 2 The presence of DR from 13 individuals with DR strains. PI: protease inhibitor; NRTI: nucleoside reverse transcriptase inhibitor; NNRTI: non-nucleoside reverse transcriptase inhibitor; ATV: atazanavir; DRV: darunavir; FPV: fosamprenavir; IDV: indinavir; LPV: lopinavir; NFV: nelfinavir; SQV: saquinavir; TPV: tipranavir/r; 3TC: lamivudine;ABC: abacavir;AZT: zidovudine;D4T: stavudine;DDI: didanosine;FTC: emtricitabine;TDF: tenofovir;EFV:efavirenz; ETR: etravirine;NVP:nevirapine;RPV:rilpivirine. 
In this study, we found that most subtypes/CRFs were present in both the heterosexually transmitted population and IDUs. This finding suggests that various HIV-1 subtypes/CRFs were disseminating between different highrisk populations via complicated transmission routes and/ or introduced by bridging populations such as female sex workers. Extensive BC recombinants were previously detected among IDUs in Dehong $[9,10,12]$. In this work, we found increased intersubtype recombinants in IDUs and the heterosexually transmitted population. In addition to $\mathrm{BC}$ recombinants, CRF01_AE and subtype $\mathrm{C}$ or $\mathrm{BC}$ recombinants were also identified for the first time in this area. Recently, extensive and complex HIV-1 recombination events between B', C and CRF01_AE were found in the neighboring northern Myanmar area [25]. Thus, the China-Myanmar border area appears to be a hot spot of active viral recombinant events. In order to effectively control the HIV epidemic in this area, comprehensive strategies including risk behavior interventions, enhanced HIV testing and proper use of ART should be emphasized.

As a linkage between the HIV-1 epidemics in Southeast Asia and China, Dehong converges many HIV-1 strains circulating in this area $[8,13,26,27]$. Dehong was also affected by the epidemic in other parts of China. For example, CRF07_BC and CRF08_BC were first detected from IDUs in Xinjiang province and Guangxi province in 1997, respectively [28,29]. However, it is believed that these two CRFs were initially formed in Yunnan Province [30] and spread through two different overland heroin trafficking routes. CRF07_BC spread northwestward to Xinjiang, and CRF08_BC eastward to Guangxi. As reported previously, these 2 CRFs were not identified in western Yunnan until $2002[9,10,12]$ but were predominant in eastern Yunnan prior to 2002, including Honghe and Wenshan prefectures [12]. Thus, it is likely that these 2 CRFs might have formed in eastern Yunnan and spread to western Yunnan. Furthermore, the epidemic could potentially influence neighboring countries if infected individuals and drug trafficking are not monitored and managed efficiently.

\section{Conclusions}

This study of HIV-1 genetic diversity and TDR-associated mutations among ART-naive individuals in Dehong, Yunnan reveals distinct subtype distribution patterns in the heterosexually transmitted population and IDUs. While subtype $C$ was the most frequently found virus in both populations, CRF01_AE was found to circulate more frequently in the heterosexually transmitted population. Strikingly, the URF recombinant events continued to rise with a prevalence second only to subtype C. The overall prevalence of TDR remained low in newly HIV-1 diagnosed and ART-naive individuals. HIV drug resistant mutations in these individuals represent a great challenge for the future of the ART program. Our findings could better guide us in the development of efficacious and effective management plans and strategies to prevent the transmission of drug resistant strains of HIV-1.

\section{Abbreviations}

DR: Drug Resistance; TDR: Transmitted Drug Resistance; ART: Antiretroviral Therapy; IDU: Injecting Drug User; PI: Protease Inhibitor; NRTI: Nucleoside Reverse Transcriptase Inhibitor; NNRTI: Non-Nucleoside Reverse Transcriptase Inhibitor; PR: Protease; RT: Reverse Transcriptase; URF: Unique Recombinant Form; CRF: Circulating Recombinant Form.

\section{Competing interests}

The authors declare that they have no competing interest.

\section{Authors' contributions}

$\mathrm{LL}$ and MJ for project initiation; MC, YM, SD, SY, YS, HL, LY and HC for data collection; MC, YM and HX for data analysis; MC, YM, AQ, LL, MJ and CYO for manuscript preparation. All authors read and approved the final manuscript.

\section{Acknowledgements}

We acknowledge the NIH Fellows Editorial Board, Serena Fuller and Marc Bulterys for editorial and manuscript preparation assistance. This work was supported by the Major Project of China's "Eleventh Five-Years Plan" for Science and Technologies Development (2009ZX10004-902 and 2008ZX10001-004) and the special grade of the financial support from China Postdoctoral Science Foundation (200902022).

\section{Author details}

${ }^{1}$ Yunnan Center for AIDS/STD Control and Prevention, Yunnan Center for Disease Control and Prevention, Kunming, Yunnan 650022, China. Department of HIV/AIDS Control and Prevention, Dehong Center for Disease Control and Prevention, Dehong, Yunnan 678400, China. ${ }^{3}$ National Center for AIDS/STD Control and Prevention, Chinese Center for Disease Control and Prevention, Beijing 102206, China. ${ }^{4}$ Laboratory of Metabolism, Center for Cancer Research National Cancer Institute, National Institutes of Health, Bethesda, MD 20892, USA. ${ }^{5}$ Global AIDS Program in China, U. S. Centers for Disease Control and Prevention, Beijing 100600, China.

Received: 23 June 2012 Accepted: 21 December 2012

Published: 28 December 2012

\section{References}

1. Beyrer C, Razak MH, Lisam K, Chen J, Lui W, Yu XF: Overland heroin trafficking routes and HIV-1 spread in south and south-east asia. AIDS 2000, 14(1):75-83.

2. Zheng X, Tian C, Choi KH, Zhang J, Cheng H, Yang X, Li D, Lin J, Qu S, Sun $X$, et al: Injecting drug use and HIV infection in southwest china. AIDS 1994, 8(8):1141-1147.

3. Duan $S$, Shen $S$, Bulterys M, Jia Y, Yang Y, Xiang L, Tian F, Lu L, Xiao Y, Wang $M$, et al: Estimation of HIV-1 incidence among five focal populations in dehong, Yunnan: a hard hit area along a major drug trafficking route. BMC Publ Health 2010, 10:180.

4. Jia Y, Sun J, Fan L, Song D, Tian S, Yang Y, Jia M, Lu L, Sun X, Zhang S, et al: Estimates of HIV prevalence in a highly endemic area of china: dehong prefecture, yunnan province. Int J Epidemiol 2008, 37(6):1287-1296.

5. Graf M, Shao Y, Zhao Q, Seidl T, Kostler J, Wolf H, Wagner R: Cloning and characterization of a virtually full-length HIV type 1 genome from a subtype B'-thai strain representing the most prevalent B-clade isolate in china. AIDS Res Hum Retroviruses 1998, 14(3):285-288.

6. Weniger BG, Takebe Y, Ou CY, Yamazaki S: The molecular epidemiology of HIV in asia. AIDS 1994, 8(Suppl 2):S13-S28.

7. Shao Y, Zhao Q, Wang B, Chen Z, Su L, Zeng Y, Zhao S, Zhang J, Duan Y, Hell W, et al: Sequence analysis of HIV env gene among HIV infected IDUs in yunnan epidemic area of china. Chin J Virol 1994, 10(4):291-299.

8. Luo CC, Tian C, Hu DJ, Kai M, Dondero T, Zheng X: HIV-1 subtype C in china. Lancet 1995, 345(8956):1051-1052. 
9. Ma YL, Zhang $Y$, Lu L, Yang L, Shi YH, Yang CJ, Luo HB, Yan WY, Min XD, Su $Y Z$, et al: The analysis of human immunodeficiency virus-1 subtypes in yunnan province. Zhonghua Yu Fang Yi Xue Za Zhi 2008, 42(12):892-894

10. Zhang Y, Lu L, Ba L, Liu L, Yang L, Jia M, Wang H, Fang Q, Shi Y, Yan W, et al: Dominance of HIV-1 subtype CRF01_AE in sexually acquired cases leads to a new epidemic in yunnan province of china. PLoS Med 2006, 3(11):e443

11. Qiu Z, Xing H, Wei M, Duan Y, Zhao Q, Xu J, Shao Y: Characterization of five nearly full-length genomes of early HIV type 1 strains in ruili city: implications for the genesis of CRF07_BC and CRF08_BC circulating in china. AIDS Res Hum Retroviruses 2005, 21(12):1051-1056.

12. Yang $R$, Xia X, Kusagawa S, Zhang C, Ben $K$, Takebe $Y$ : On-going generation of multiple forms of HIV-1 intersubtype recombinants in the yunnan province of china. AIDS 2002, 16(10):1401-1407.

13. Cheng H, Zhang J, Capizzi J, Young NL, Mastro TD: HIV-1 subtype E in yunnan, china. Lancet 1994, 344(8927):953-954.

14. Bartlett JA, DeMasi R, Quinn J, Moxham C, Rousseau F: Overview of the effectiveness of triple combination therapy in antiretroviral-naive HIV-1 infected adults. AIDS 2001, 15(11):1369-1377.

15. Palella FJ Jr, Delaney KM, Moorman AC, Loveless MO, Fuhrer J, Satten GA, Aschman DJ, Holmberg SD: Declining morbidity and mortality among patients with advanced human immunodeficiency virus infection. HIV outpatient study investigators. N Engl J Med 1998, 338(13):853-860.

16. Tamalet C, Fantini J, Tourres C, Yahi N: Resistance of HIV-1 to multiple antiretroviral drugs in france: a 6-year survey (1997-2002) based on an analysis of over 7000 genotypes. AIDS 2003, 17(16):2383-2388.

17. Li JY, Li HP, Li L, Li H, Wang Z, Yang K, Bao ZY, Zhuang DM, Liu SY, Liu YJ, et al: Prevalence and evolution of drug resistance HIV-1 variants in henan, china. Cell Res 2005, 15(11-12):843-849.

18. Bennett DE, Camacho RJ, Otelea D, Kuritzkes DR, Fleury H, Kiuchi M, Heneine W, Kantor R, Jordan MR, Schapiro JM, et al: Drug resistance mutations for surveillance of transmitted HIV-1 drug-resistance: 2009 update. PLoS One 2009, 4(3):e4724.

19. de Oliveira T, Deforche K, Cassol S, Salminen M, Paraskevis D, Seebregts C, Snoeck J, van Rensburg EJ, Wensing AM, van de Vijver DA, et al: An automated genotyping system for analysis of HIV-1 and other microbial sequences. Bioinformatics 2005, 21(19):3797-3800.

20. Jeannot AC, Reigadas S, Schrive MH, Pinson P, Fleury HJ: Pol bootscanning analysis of HIV type 1 can exhibit unexpected recombinations. AIDS Res Hum Retroviruses 2009, 25(7):713-716.

21. Lu L, Jia M, Ma Y, Yang L, Chen Z, Ho DD, Jiang Y, Zhang L: The changing face of HIV in china. Nature 2008, 455(7213):609-611.

22. Bennett DE, Bertagnolio S, Sutherland D, Gilks CF: The world health Organization's global strategy for prevention and assessment of HIV drug resistance. Antivir Ther 2008, 13(Suppl 2):1-13.

23. Wang MJ, Duan S, Xiang LF, Yang YC, Tu YQ, Wang JB, Yao J, Qi J, Jiang Y: Study on the drug resistance situation among recently infected HIV-1 patients in dehong. Zhonghua Liu Xing Bing Xue Za Zhi 2008, 29(9):905-908

24. Yang S, Fan Y, Li H, Yang B, Li J, Gao L, Zhong M, Lei S, Zhou Z: Investigation of pol gen variation of HIV-1 epidemic strains after treatment with HARRT at dehong prefecture and kunming in yunnan province. Chin J Lab Med 2011, 34(4):315-320.

25. Pang W, Zhang C, Duo L, Zhou YH, Yao ZH, Liu FL, Li H, Tu YQ, Zheng YT: Extensive and complex HIV-1 recombination between $\mathrm{B}^{\prime}, \mathrm{C}$ and CRF01_AE among IDUs in Northern Myanmar. AIDS 2012, 26(9):1121-1129.

26. Motomura K, Kusagawa S, Lwin HH, Thwe M, Kato K, Oishi K, Yamamoto N, Zaw M, Nagatake T, Takebe Y: Different subtype distributions in two cities in myanmar: evidence for independent clusters of HIV-1 transmission. AIDS 2003, 17(4):633-636

27. Kusagawa S, Sato H, Watanabe S, Nohtomi K, Kato K, Shino T, Thwe M, Oo KY, Lwin S, Mra R, et al: Genetic and serologic characterization of HIV type 1 prevailing in myanmar (burma). AIDS Res Hum Retroviruses 1998, 14(15):1379-1385.

28. Su L, Graf $M$, Zhang $Y$, von Briesen $H$, Xing $H$, Kostler J, Melzl $H$, Wolf $H$, Shao $Y$, Wagner R: Characterization of a virtually full-length human immunodeficiency virus type 1 genome of a prevalent intersubtype (C/B') recombinant strain in China. J Virol 2000, 74(23):11367-11376.

29. Piyasirisilp S, McCutchan FE, Carr JK, Sanders-Buell E, Liu W, Chen J, Wagner $\mathrm{R}$, Wolf $\mathrm{H}$, Shao $\mathrm{Y}$, Lai $\mathrm{S}$, et al: A recent outbreak of human immunodeficiency virus type 1 infection in southern china was initiated by two highly homogeneous, geographically separated strains, circulating recombinant form $\mathrm{AE}$ and a novel $\mathrm{BC}$ recombinant. J Virol 2000, 74(23):11286-11295.

30. Tee KK, Pybus OG, Li XJ, Han X, Shang H, Kamarulzaman A, Takebe Y: Temporal and spatial dynamics of human immunodeficiency virus type 1 circulating recombinant forms 08_BC and 07_BC in asia. J Virol 2008, 82(18):9206-9215.

doi:10.1186/1471-2334-12-382

Cite this article as: Chen et al:: Genetic diversity and drug resistance among newly diagnosed and antiretroviral treatment-naive HIV-infected individuals in western Yunnan: a hot area of viral recombination in China. BMC Infectious Diseases 2012 12:382.

\section{Submit your next manuscript to BioMed Central and take full advantage of:}

- Convenient online submission

- Thorough peer review

- No space constraints or color figure charges

- Immediate publication on acceptance

- Inclusion in PubMed, CAS, Scopus and Google Scholar

- Research which is freely available for redistribution

Submit your manuscript at www.biomedcentral.com/submit
C) Biomed Central 